\title{
ISCEV guidelines for clinical multifocal electroretinography (2007 edition)
}

\author{
Donald C. Hood · Michael Bach · Mitchell Brigell · David Keating • \\ Mineo Kondo · Jonathan S. Lyons · Anja M. Palmowski-Wolfe
}

Received: 2 October 2007/Accepted: 4 October 2007/Published online: 31 October 2007

(C) Springer-Verlag 2007

\begin{abstract}
The clinical multifocal electroretinogram (mfERG) is an electrophysiological test of local retinal function. With this technique, many local ERG responses, typically 61 or 103 , are recorded from the cone-driven retina under light-adapted conditions. This document specifies guidelines for
\end{abstract}

The authors belongs to International Society for Clinical Electrophysiology of Vision.

\section{C. Hood (凶)}

Departments of Psychology and Ophthalmology,

Columbia University, New York, NY, USA

e-mail:dch3@columbia.edu

\section{Bach}

Univ.- Augenklinik, Freiburg, Germany

\section{Brigell}

Translational Medicine, Novartis Institutes for

Biomedical Research, Cambridge, MA, USA

D. Keating

Department of Ophthalmology, Gartnavel General

Hospital, Glasgow, UK

M. Kondo

Department of Ophthalmology, Nagoya University

School of Medicine, Nagoya, Japan

\section{J. S. Lyons}

Washington Hospital Center Program in Ophthalmology, Georgetown University, Silver Spring, MD, USA

A. M. Palmowski-Wolfe

Eye Hospital, University of Basel, Basel, Switzerland performance of the test. It also provides detailed guidance on technical and practical issues, as well as on reporting test results. The main objective of the guidelines is to promote consistent quality of mfERG testing and reporting within and among centers. These 2007 guidelines, from the International Society for Clinical Electrophysiology of Vision (ISCEV: http://www.iscev.org), replace the ISCEV guidelines for the mfERG published in 2003.

Keywords Clinical guidelines - Electroretinogram · Multifocal electroretinogram
Abbreviations
CRT Cathode ray tube
ERG Electroretinogram
ISCEV International Society for Clinical Electrophysiology of Vision
mfERG Multifocal electroretinogram
PERG Pattern electroretinogram

\section{Introduction}

The electroretinogram (ERG) is a mass potential, the result of the summed electrical activity of the cells of the retina. Full-field electroretinography is a 
well-established clinical technique for evaluating global retinal function [1]. The multifocal ERG (mfERG) technique was developed to provide a topographic measure of retinal electrophysiological activity. With this technique, many local ERG responses, typically 61 or 103 , are recorded from the cone-driven retina under light-adapted conditions. In 2003, the International Society for Clinical Electrophysiology of Vision (ISCEV) published guidelines for recording the mfERG [2]. These were guidelines, not standards, to allow for further research before standards were set.

Although the mfERG now has been used for more than 10 years to aid in the diagnosis of diseases of the retina, ISCEV decided that it is still premature to set specific standards. However, to take into consideration recent developments in technology and practice, this document provides revised guidelines for recording clinical mfERGs. These guidelines will be reviewed periodically, consistent with ISCEV's practice.

\section{Description of multifocal electroretinography}

The mfERG technique is a method of recording local electrophysiologic responses from different regions of the retina. Electrical responses from the eye are recorded with a corneal electrode as in conventional, full-field ERG recording. However, the nature of the stimulus and the form of the analysis differ. These differences allow a topographic map of local ERG activity to be measured. For the basic mfERG described here, the retina is stimulated with an array of hexagonal elements, each of which has a $50 \%$ chance of being illuminated every time the frame changes (Fig. 1). Although the pattern appears to flicker randomly, each element follows the same pseudo-random sequence of illumination with the starting point displaced in time relative to other elements. By correlating the continuous ERG signal with the sequence of on- and off-phases of each element, the local ERG signal is calculated. Although we will refer to these local ERG signals as mfERG responses, it is important to keep in mind that they are not direct electrical potentials from local regions of retina, but rather they are a mathematical extraction of the signal. Further, because the stimulation rate is rapid, the waveform of the local mfERG response can be influenced both by preceding ('adaptation effects') and subsequent stimuli ('induced effects'), as well as by the responses to light scattered on other retinal areas.

The typical waveform of the basic mfERG response (also called the first-order response or first-order kernel) is a biphasic wave with an initial negative deflection followed by a positive peak (Fig. 2). There is usually a second negative deflection after the positive peak. These three peaks are called N1, P1 and N2, respectively. There is evidence that $\mathrm{N} 1$ includes contributions from the same cells that contribute to the a-wave of the full-field cone ERG, and that P1 includes contributions from the cells contributing to the cone b-wave and oscillatory potentials. Although there is some homology between the mfERG waveform and the conventional ERG, the stimulation rates are higher for the mfERG and, as noted above, the mfERG responses are mathematical extractions. Thus, the mfERG responses are not technically "little ERG responses". Therefore, the designations 'a-wave' and 'b-wave', used for the fullfield ERG, are not appropriate to describe features of the mfERG waveform.
Fig. 1 (a) Representative hexagonal mfERG stimulus array with 61 elements scaled with eccentricity. Roughly half of the elements are illuminated at any one time. (b) Same as in panel A for an array with 103 elements
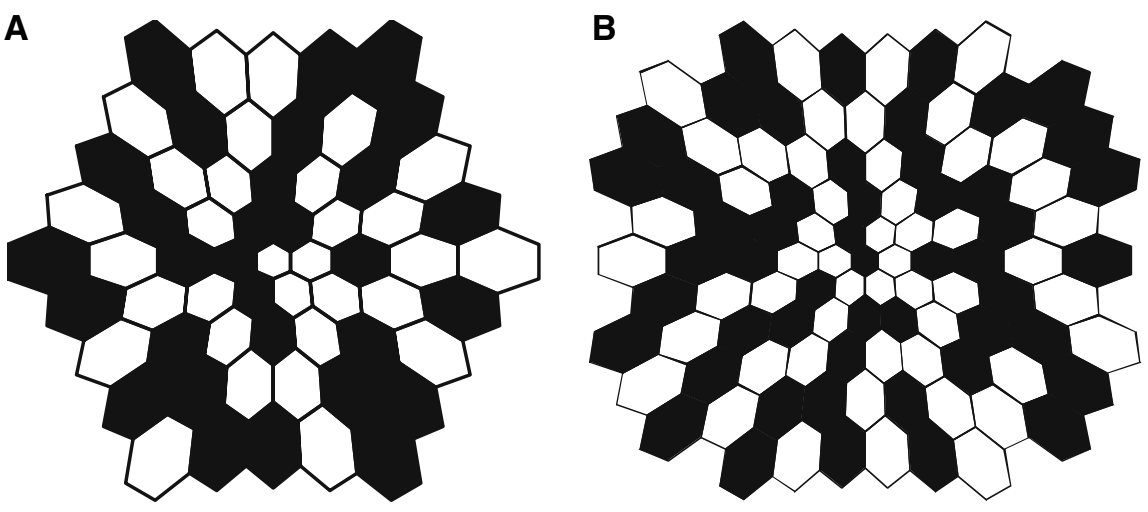


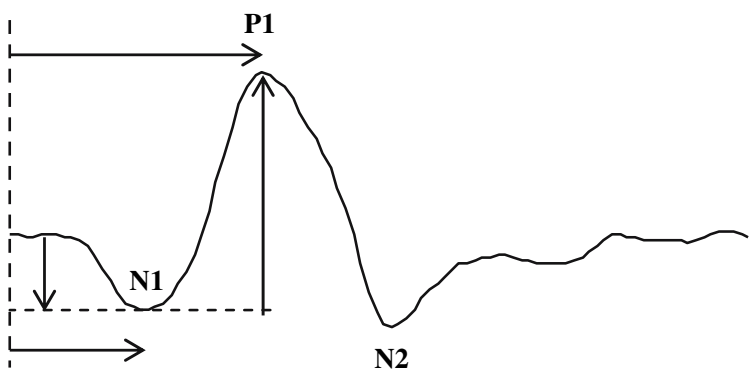

Fig. 2 Diagram of an mfERG response to show the designation of the major features of the waveform

\section{Basic technology}

Electrodes

\section{Recording electrodes}

Poor or unstable electrode contact is a major cause of poor quality records. It is important to follow the recommendations concerning fiber, foil, loop and contact lens electrodes in the full-field ERG Standard [1] and Pattern ERG (PERG) Standard [3]. In particular, electrodes that contact the cornea, or nearby bulbar conjunctiva, are required. In addition, good retinal image quality and proper refraction is desirable.

\section{Reference and ground electrodes}

Proper application of suitably conductive electrodes is essential for reliable mfERG recordings. Follow the recommendations made in other ISCEV Standards $[1,3]$.

\section{Electrode characteristics, stability and cleaning}

Follow the recommendations in the ISCEV full-field ERG and/or PERG Standards [1, 3].

\section{Stimulation}

\section{Stimulus source}

Until recently, the mfERG stimuli were most commonly displayed on a cathode ray tube (CRT), i.e. a monitor. CRT monitors are being rapidly replaced with other devices such as liquid crystal display (LCD) projectors, arrays of light emitting diodes (LEDs) and organic LEDs (OLEDs). These alternative modes of stimulation can affect the amplitude and waveform of the mfERG making it essential to specify the type of display when reporting results. ${ }^{1}$

\section{Frame frequency}

A CRT frame frequency of $75 \mathrm{~Hz}$ has been used widely. (LCD displays use $60 \mathrm{~Hz}$.) Use of different frequencies can substantially alter the amplitude and waveform of the mfERG response. Whatever frame frequency is used, normative values for normal healthy subjects need to be determined separately for that frequency. Further, it is essential to specify the frame frequency when reporting results.

\section{Luminance and contrast}

In general, the luminance of the stimulus elements for CRT displays should be $100-200 \mathrm{~cd} / \mathrm{m}^{2}$ in the lighted state and low enough in the dark state to achieve a contrast of $\geq 90 \%$. This means that the mean screen luminance during testing will be $50-100 \mathrm{~cd} / \mathrm{m}^{2}$. Although higher luminance levels can be used, the ability to detect local defects may be decreased due to the effects of stray light. In addition, the luminance requirements may differ if a non-CRT display is used. In any case, the surround region of the display (the area beyond the stimulus hexagons) should have a luminance equal to the mean luminance of the stimulus array.

\section{Calibration}

As with other electrophysiologic signals, luminance and contrast affect the recorded signal and it is

\footnotetext{
${ }^{1}$ The 'response time' is particularly important and must be sufficiently brief. (By response time we mean the amount of time a local element (e.g. pixel) takes to go from "black" to "white" and back to "black" again.) CRT monitors typically have response times under $2 \mathrm{~ms}$, whereas the response times of some of the older LCD displays can be as long as $25 \mathrm{~ms}$. Response times should be considerably less than the frame interval (e.g. $<<13.33 \mathrm{~ms}$ for a frame rate of $75 \mathrm{~Hz}$ ).
} 
important to calibrate the stimulus following ISCEV guidelines [4]. The luminance of the dark and the light stimulus elements should be measured with an appropriate calibrator or spot meter. Many monitor screens are not of uniform brightness over the entire screen. While some variation is to be expected, a variation of greater than $15 \%$ is considered unacceptable. Some commercial systems are equipped to calibrate the display. If this ability is not present, we urge manufacturers to provide instructions for calibration of their devices.

\section{Stimulus parameters}

Stimulus pattern: The typical mfERG display is a hexagonal stimulus pattern scaled in size to produce mfERG responses of approximately equal amplitude across the retina. Thus, the central hexagons are smaller than the more peripheral ones. Different patterns may be useful in special cases (e.g. equal size hexagons for patients with eccentric fixation). These guidelines cover only the typical stimulus pattern, scaled to produce approximately equal size responses for healthy control subjects.

Flicker sequence: Most commercial mfERG instruments use an m-sequence to control the temporal sequence (between light and dark) of the stimulus elements. An m-sequence, in which the elements can change with every frame, is recommended for routine testing. Different sequences, or the inclusion of global light or dark frames, have been suggested for specialized applications, but they are not a part of these guidelines, and should not be done in exclusion of a "standard" mfERG for routine clinical purposes.

Stimulus size and number of elements: The overall stimulus pattern should subtend a visual angle of 20 $30^{\circ}$ on either side of the fixation point. The stimulus region can be divided into different numbers of hexagons. The most frequently used patterns have 61 or 103 hexagons, with 241 hexagons occasionally used. The choice depends on balancing the need for good spatial resolution and a high signal-to-noise ratio, while minimizing the recording time. (See discussion below under clinical protocol).

Fixation targets: Stable fixation is essential for obtaining reliable mfERG recordings. Central fixation dots, crosses and circles are available with most commercial systems. The fixation targets should cover as little of the central stimulus element as possible to avoid diminishing the response. However, the examiner should always verify that the patient can see the fixation target. When the fixation targets are enlarged for low-vision patients, care should be taken not to obscure regions of interest. For example, a larger central cross will lead to a smaller central response simply due to occlusion of more of the stimulus.

Recording, analyzing and presenting results

\section{Amplifiers and filters}

The gain of the amplifier should produce recognizable signals without saturation. Appropriate bandpass filtering removes extraneous electrical noise, without distorting waveforms of interest. For a "standard" mfERG, the high pass cutoff can range between 3 and $10 \mathrm{~Hz}$ and the low pass cutoff between 100 and $300 \mathrm{~Hz}$. Filter settings, even within the ranges suggested, will influence the response waveform. Thus, the filter settings should be the same for all subjects tested by a given laboratory, as well as for the norms to which they are compared. Linefrequency or notch filters should be avoided.

\section{Signal analysis}

Artifact rejection: Because blinks and other movements can distort the recorded waveforms, commercial software usually includes 'artifact rejection' algorithms to eliminate some of these distortions. When applying an artifact rejection procedure after the recording, care should be exercised to assure that clinically important aspects of the waveform are not being modified. In any case, artifact rejection procedures should be specified when reporting mfERG results.

Spatial averaging: In order to reduce noise and smooth the waveforms, some commercial programs allow the averaging of the response from each stimulus element with a percentage of the signal from the neighboring elements. Spatial averaging can help visualize the mfERG signal in noisy records. However, spatial averaging may obscure small, local changes or the borders of regions of dysfunction. 
Thus, it should be used with care and specified when reporting results. Further, the default conditions of commercial software should be examined, as spatial averaging may be a default condition.

\section{Displaying results}

Trace arrays: All commercial programs can produce an array of the mfERG traces (Fig. 3a, b). This display of the results is useful for visualizing areas of abnormality and for comparing the mfERG results to visual fields from perimetry. The trace array is the basic mfERG display and should always be included in the report of clinical results. Commercial software typically allows for these responses to be displayed with either a visual field view or retinal view as the frame of reference. The frame of reference should be indicated. Note that in Fig. 3, each of the 61 (panel a) or 103 (panel b) responses are shown in approximately correct topographic representation. Adding contours to show the loci of different eccentricities, as in Fig. 4, can help in determining whether the regions of low amplitude correspond to regions of the visual field that show a loss of sensitivity.

Group averages: Commercial programs allow for the averaging of groups of responses from the trace arrays. This can be helpful for comparing quadrants, hemiretinal areas, normal and abnormal regions of two eyes, or successive rings from center to periphery. The latter is particularly useful for patients who have diseases that produce approximately radially symmetric dysfunction. In addition, responses from stimulus elements associated with a local area of interest can be averaged for comparison with a similar area in an unaffected eye or to data from control subjects. Figure 5 shows the result of grouping the responses in Fig. $3 \mathrm{~b}$ by rings. The most commonly used display is response density (Fig. 5a), in which the responses from the elements in each ring are summed and then divided by the area of these elements. Figure $5 \mathrm{~b}$ and $\mathrm{c}$ are alternative representations of the data in which summed responses from
Fig. 3 Sample mfERG trace arrays (field view) with 61 elements (panel a) and 103 elements (panel b). (c, d) The 3-D response density plots (field view) associated with panels $\mathbf{a}$ and $\mathbf{b}$
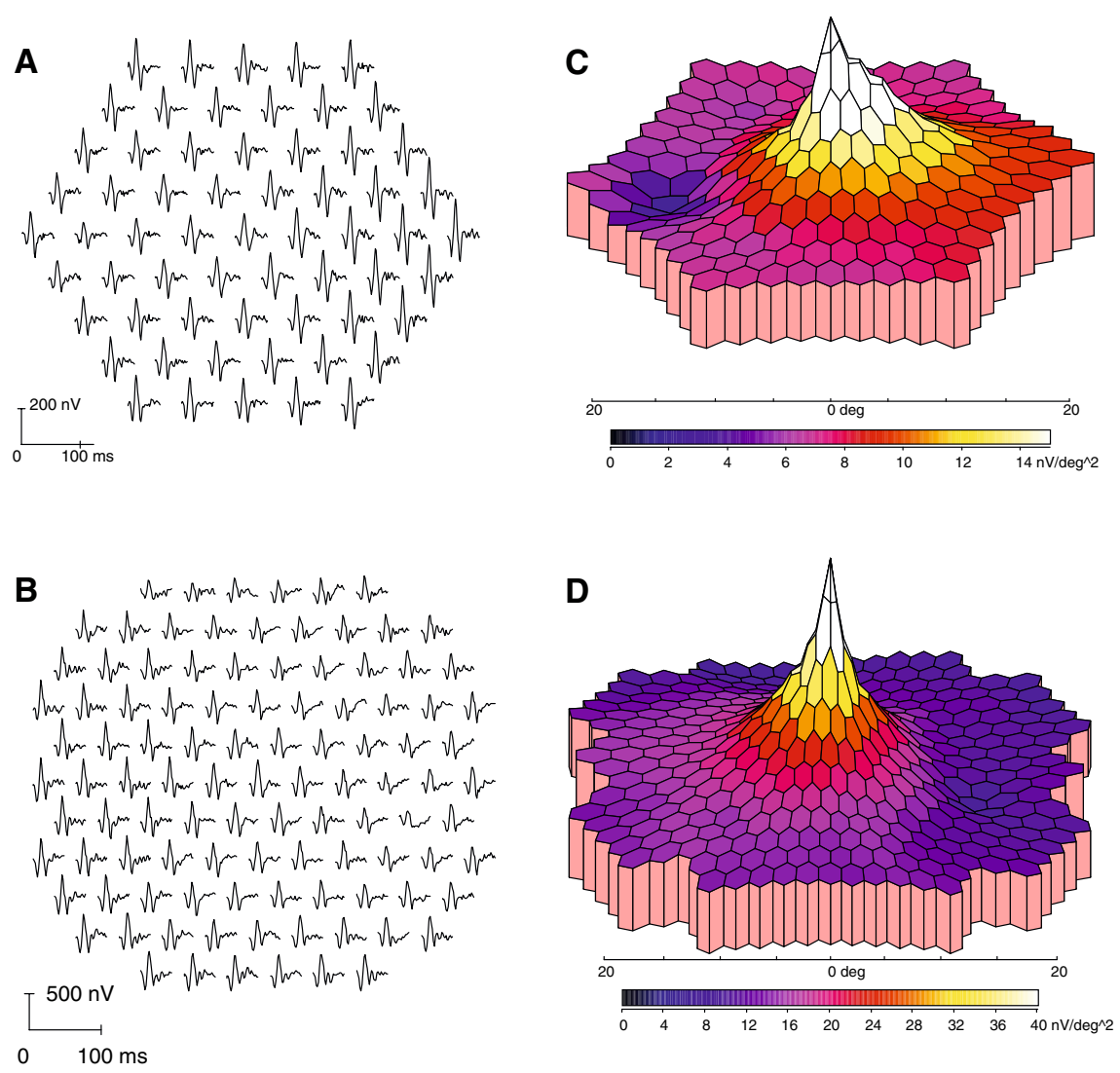
Fig. 4 The mfERG trace array (left panel, field view) and the probability plot from standard automated perimetry (right panel) for a patient with retinitis pigmentosa. The contours for a radius of 5 and $15^{\circ}$ are shown. The light gray, dark gray, and black squares indicate statistically significant field loss at the 5,1 and 0.5 percent levels, respectively

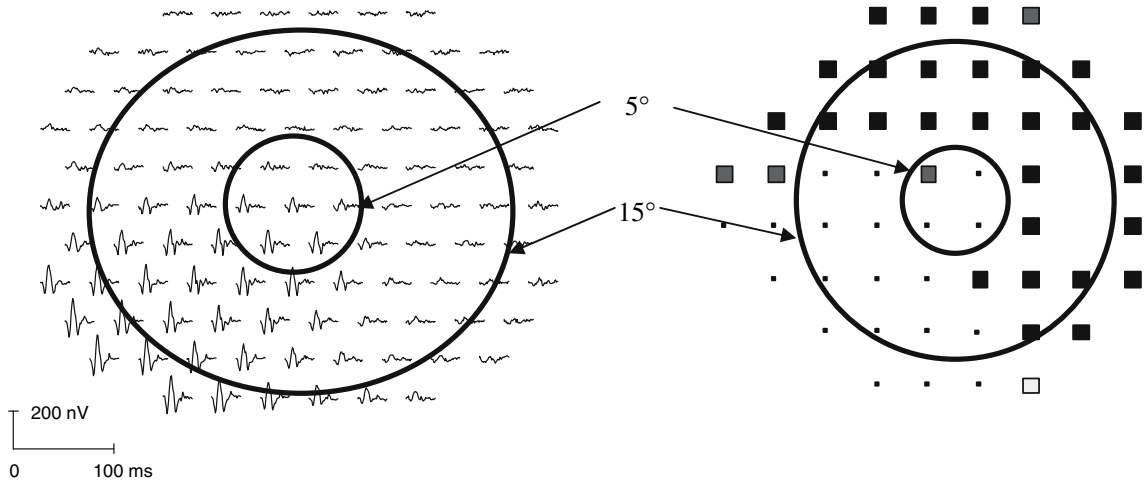

each ring are shown (panel c) or are normalized so that they have the same amplitude (panel b).

Topographic (3-D) response density plots: The 3D plot (Fig. 3c, d) shows the overall signal strength per unit area of retina. This display can sometimes be useful for illustrating certain types of pathology. In addition, the quality of fixation can be assessed by observing the location and depth of the blind spot. However, there are major dangers in using the 3-D plot in assessing retinal damage. First, information about the waveforms is lost. Thus large, but abnormal, or delayed responses can produce normal 3-D plots. Second, a central peak in the 3-D plot can be seen in some records without any retinal signal (see,
Appendix: Artifact recognition examples for electrical noise and weak signals). Finally, the appearance of the 3-D plot from a given recording is dependent on how the local amplitude is measured. Whenever 3-D plots are presented, the method used to measure local amplitude should be identified, and the corresponding mfERG trace array also should be presented.

Signal extraction: Kernels-This document is aimed at the general mfERG user and only describes the basic response, the first-order kernel. Higher order kernels, particularly the second-order kernel, occasionally are reported, and used in special applications.
Fig. 5 The mfERG responses in Fig. $3 b$ were grouped by concentric rings and summed to yield the 'Summed' responses in panel c. These summed responses are divided by the area of the elements of the ring for the 'Response Density' responses (panel a) and normalized so each has the same amplitude for the 'Normalized' responses (panel b)
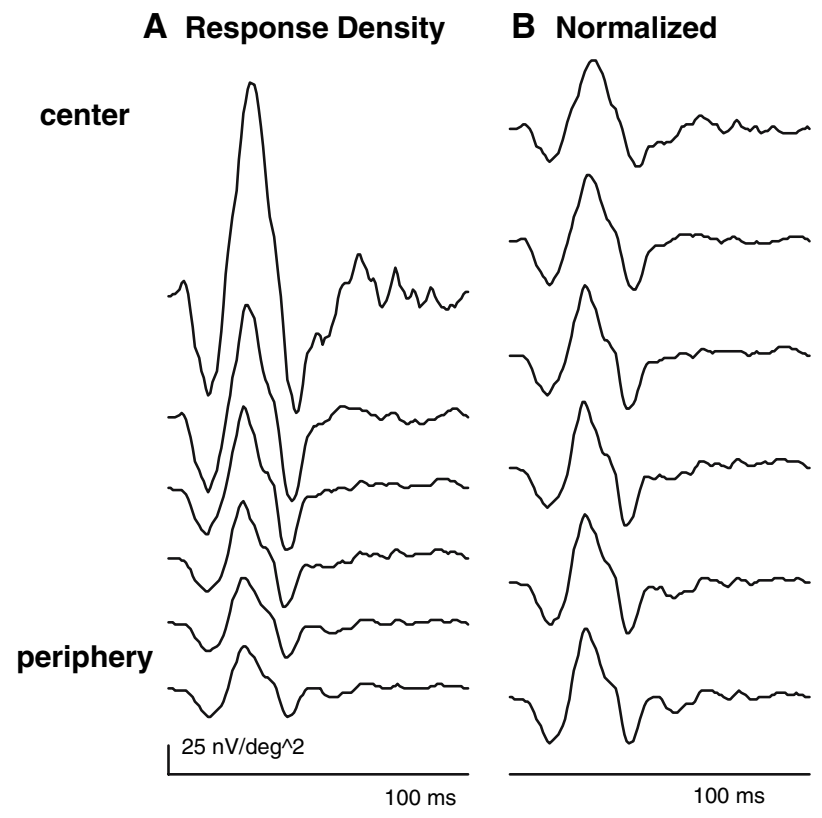

C Summed

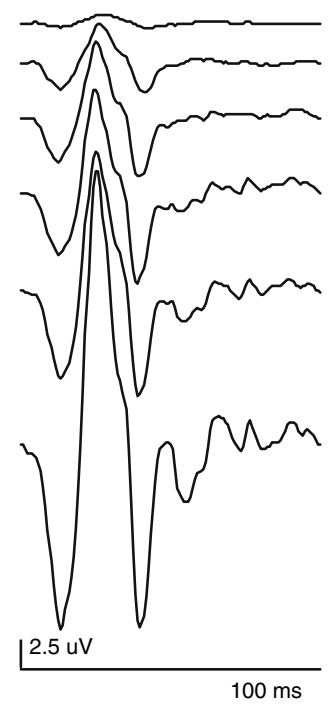




\section{Clinical protocol}

Patient preparation

Pupils

The pupils should be fully dilated and pupil size noted.

\section{Patient positioning}

Subjects should sit comfortably in front of the screen. Relaxation of facial and neck muscles will reduce artifacts from muscles; a headrest may be helpful. The appropriate viewing distance will vary with screen size, in order to control the area (visual angle) of retina being stimulated.

\section{Fixation monitoring}

Good fixation, both central and steady, is essential. Thus, fixation should be monitored, preferably by the use of monitoring instrumentation available on some units. When this option is not available, careful direct observation may be employed.

\section{Refraction}

Although there is some evidence that the mfERG is unaffected by moderate blurring of the retinal image in healthy individuals, we recommend refraction for optimal acuity. On some commercial machines, a manual adjustment of the viewing optics is possible. Alternatively, lenses can be placed in a holder positioned in front of the eye. In the latter case, the viewing distance must be adjusted to compensate for the relative magnification of the stimulus. Also care must be taken to avoid blocking the view of the stimulus screen by the rim of the lens or the lens holder and thus creating an apparent scotoma.

\section{Monocular versus binocular recording}

Recording is typically done with monocular stimulation. Those who record binocularly should be aware that signals can be altered by misalignment of the eyes.

\section{Adaptation}

Pre-adaptation (before test): Subjects should be exposed to ordinary room lighting for at least $15 \mathrm{~min}$ prior to testing. Longer adaptation times may be needed after exposure to bright sun or bright lights such as those used for fundus photography or retinoscopy.

Room illumination: Moderate or dim room lights should be on and ideally should produce illumination close to that of the stimulus screen.

Stimulus and recording parameters

\section{Stimulus Size}

The stimulus should subtend $20-30^{\circ}$ of visual angle on either side of the fixation point.

\section{Number of elements}

A display containing 61 or 103 elements should be used.

\section{Duration of recording}

A total recording time of at least $4 \mathrm{~min}$ for 61 element arrays, or $8 \mathrm{~min}$ for 103 element arrays, is recommended, although these times might be adjusted by experienced laboratories according to clinical needs. The overall recording time is divided into shorter segments (e.g. 15-30 s) so that subjects can rest between runs if necessary and also so that a poor record (from noise, movement or other artifacts) can be discarded and repeated without losing prior data.

\section{Trade-offs}

Various manipulations will affect the signal-to-noise ratio (SNR) of the responses. In particular, decreasing 
the size (increasing the number for a fixed stimulus field size) of the stimulus elements and decreasing the duration of the recording will decrease the SNR of the responses. While decreasing the number (increasing the size) of elements will increase the SNR, it will decrease the spatial resolution of the test. In general, conditions with larger (i.e. 61) elements and a shorter recording time (e.g. $4 \mathrm{~min}$ ) are easier for the patient and suitable for a general screening of macular function. On the other hand, conditions with 103 elements and a longer recording time (e.g. $8 \mathrm{~min}$ ) are useful for assessing foveal function and mapping the outline of retinal defects. Very small elements (such as a 241 hexagon array) may sometimes be helpful for diseases with very small or irregular effects. Repeat recording is recommended to confirm small or subtle abnormalities.

Further, the choice of electrode type will also influence the SNR of the responses. For example, bipolar corneal contact electrodes yield recordings with the highest SNR. Thus, longer recording times, repeat measurements and/or fewer stimulus elements are necessary to obtain comparable SNRs when a foil or fiber electrode is used.

\section{Data reporting}

\section{Mode of display}

Trace arrays: It is essential to show the trace array when reporting mfERG results (see Fig. 3a, b). These arrays not only show topographic variations, but also demonstrate the quality of the records, which is important in judging the validity of any suspected variations from normal. Trace lengths of $100 \mathrm{~ms}$ or more should be used for these displays. (It is hard to detect interference from line frequency and/or kernel overlap in shorter trace lengths.).

Group averages: Arranging responses by groups can be a useful way to summarize the data. Concentric rings of traces, from the center outward, are most commonly used. Regions with fundus pathology can be averaged together if desired. Most laboratories report response density (Fig. 5a).

Three-dimensional plots: These should be used with caution and only when accompanied by trace arrays (see above). The 3-D plots (Fig. 3c, d), without accompanying trace arrays, can be misleading (see
Appendix). Note that if fixation is steady and central, a clear depression due to the blindspot should be present and located in the appropriate place.

Measurements calibration marks: Calibration marks must accompany all traces or graphs. This will enable comparisons among patients or within a patient on sequential visits.

Measuring mfERG amplitude and timing: The N1 response amplitude is measured from the starting baseline to the base of the N1 trough; the P1 response amplitude is measured from the $\mathrm{N} 1$ trough to the P1 peak (see Fig. 2). The peak times (implicit times) of $\mathrm{N} 1$ and $\mathrm{P} 1$ are measured from the stimulus onset. Measurements of group averages should routinely include the $\mathrm{N} 1$ and $\mathrm{P} 1$ amplitudes and peak times.

Commercial software provides measures of the overall amplitude and timing of the mfERG traces. There are various procedures for measuring amplitude (e.g. trough-to-peak amplitude), latency (e.g. response shifting, response stretching, time to peak), or overall response waveform (e.g. scalar product, root-mean-square (RMS)). A description of these techniques is beyond the scope of these guidelines. However, it should be noted that when a template is needed (e.g. for scalar product measures), the template should be formed from age-similar control data obtained from that laboratory.

Color scales: The use of color scales is optional; care should be taken when reproducing color images on a gray scale as the luminance sequences may not be in the proper order.

\section{Normal values}

Each laboratory must develop its own normative data. Variations in recording equipment and parameters make the use of data from other sources inappropriate. Because electrophysiologic data are not necessarily described by a normal distribution, laboratories should report the median value rather than the mean, and determine boundaries of normality. The mfERG, like the full-field ERG, is somewhat smaller in amplitude in older individuals and in those with highly myopic eyes. Although these effects are generally not large, they can be important in the evaluation of some patients. In any case, age-adjusted normative data is recommended. 


\section{Reporting of artifacts and their resolution}

Reports should indicate any problems with the recording such as movement, head tilt, poor refraction capability, poor fixation, etc. that might affect reliability and interpretation. Also, indicate explicitly any artifact reduction procedures or post-processing maneuvers used to prepare the data. This should include the type and number of artifact rejection steps, the spatial averaging with neighbors (noting the extent and number of iterations), and any other averaging or filtering procedures.

Acknowledgements This document was approved by ISCEV at the August, 2007 meeting in Hyderabad, India. We thank Drs. Colin Barber, Marc Bearse, Anne Fulton, Laura Frishman, Karen Holopigian, Ulrich Kellner, Michael Marmor, Daphne McCulloch and Thomas Wright for their contributions to this document.

\section{Appendix: artifacts in mfERG recordings}

There are a number of artifacts that can complicate the recording or interpretation of the mfERG. We list and illustrate some of the more common ones below, along with brief suggestions for the avoidance and/or correction of these problems.

Common types of artifact

Electrical noise (Fig. A1)

Poor electrode contacts, poor grounding or ambient sources of noise can cause line current $(50$ or $60 \mathrm{~Hz})$

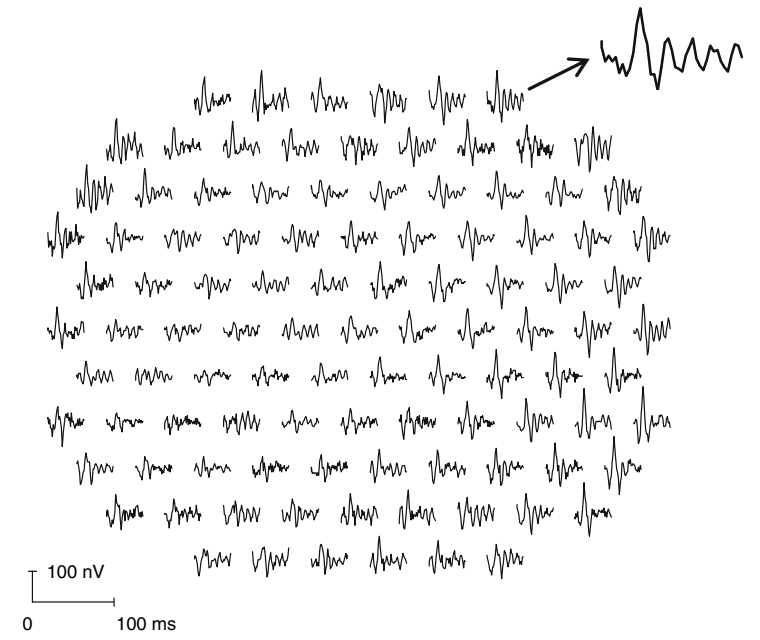

Fig. A1 Electrical noise. The trace array shows $60 \mathrm{~Hz}$ signals contaminating the responses or CRT monitor (e.g. $75 \mathrm{~Hz}$ ) interference that alters the mfERG traces. Noise is usually easy to identify in trace arrays if the time epoch of the trace is sufficiently long. Solution: Better electrode contact, grounding and/or electrical shielding.

\section{Movement errors}

Large eye movements can produce saturation of the amplifiers as well as aberrant drifting or fluctuation in the signal recorded. Milder degrees of eye movement, or unsteady fixation, can cause a smearing of the responses among different loci, and thus reduce the resolution, making it difficult to detect small areas of dysfunction. If the blind spot is not visible in the 3-D density plot, this may be a clue to poor fixation. Solution: Observe the amount of noise during the recording. Contaminated runs or segments should be
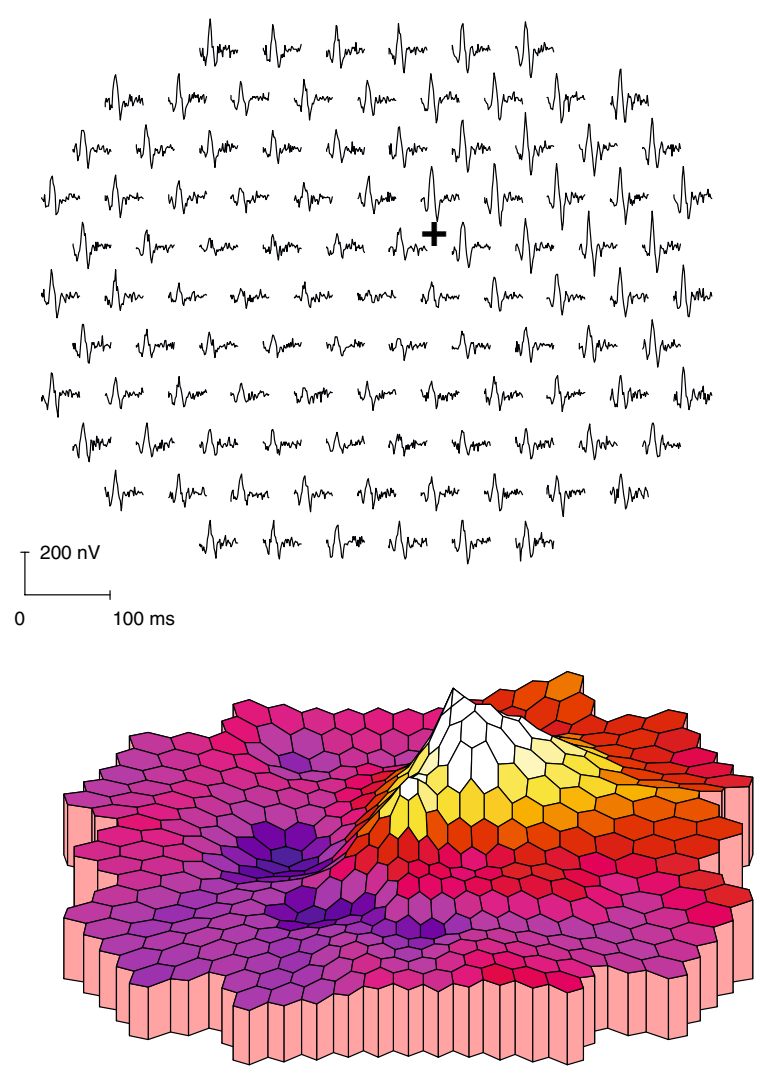

Fig. A2 Eccentric fixation. The subject with normal vision fixated at the + instead of at the center. As a result, the calculated response magnitudes are altered, and there is a false appearance of central retinal dysfunction 
discarded and re-recorded. The monitoring and control of fixation should be improved.

\section{Eccentric fixation (Fig. A2)}

Eccentric fixation can cause trace arrays and topographic 3-D plots that are depressed centrally, or show a 'sloping' appearance with low signals on one side and high on the other. Solution: Check fixation, or use a special low vision fixation target.

\section{Orientation/shadowing errors (Fig. A3)}

Shadowing appears when a subject is poorly centered or when either the refraction lens or the recording contact lens is blocking some of the display. The trace arrays and 3-D plots show depression in one part of the array and sometimes an elevation on the opposite side. These errors must be distinguished from patterns of disease, and from the small normal nasal-temporal variation. Solution: Center the lenses

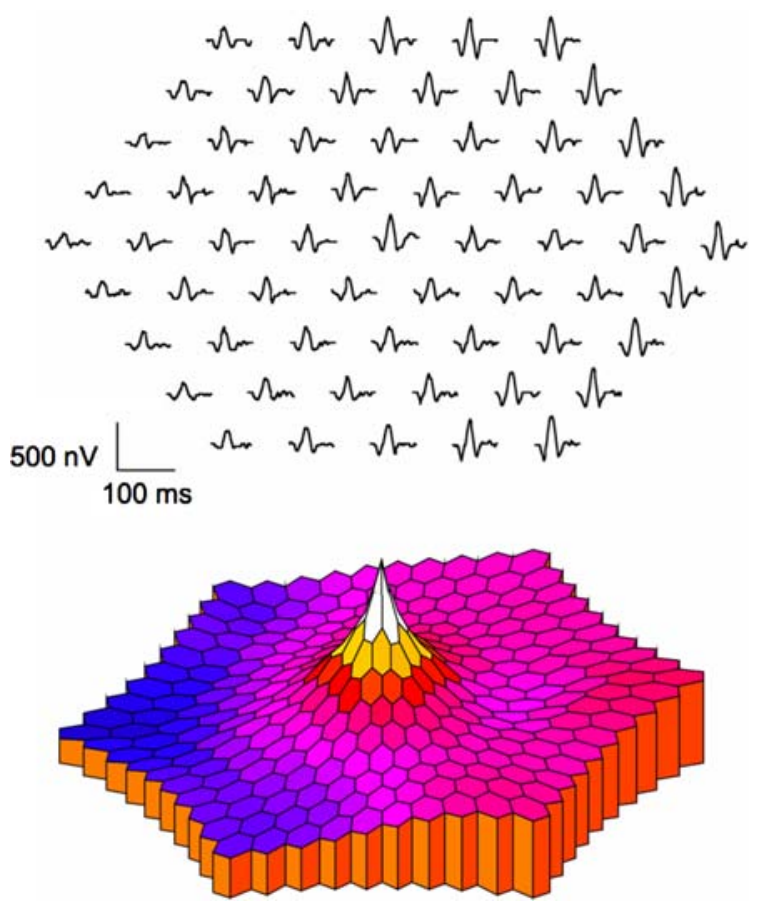

Fig. A3 Shadowing error. The subject's view was obscured on one side by the edge of a refracting lens. As a result, both the trace array and 3-D plot show a false reduction in amplitude on one side and subject, place the refracting lens close to the eye and monitor eye position.

\section{Erroneous central peak (Weak signal artifact) (Fig. A4)}

When using a display of scaled elements (Fig. 1), 3-D density plots can show a central peak, even when no signal is present. This occurs because the response amplitude (signal plus noise) is divided by the area of the hexagon. The effects of noise are magnified in the center where the overall amplitude is divided by a small area. Solution: Look at the trace array to
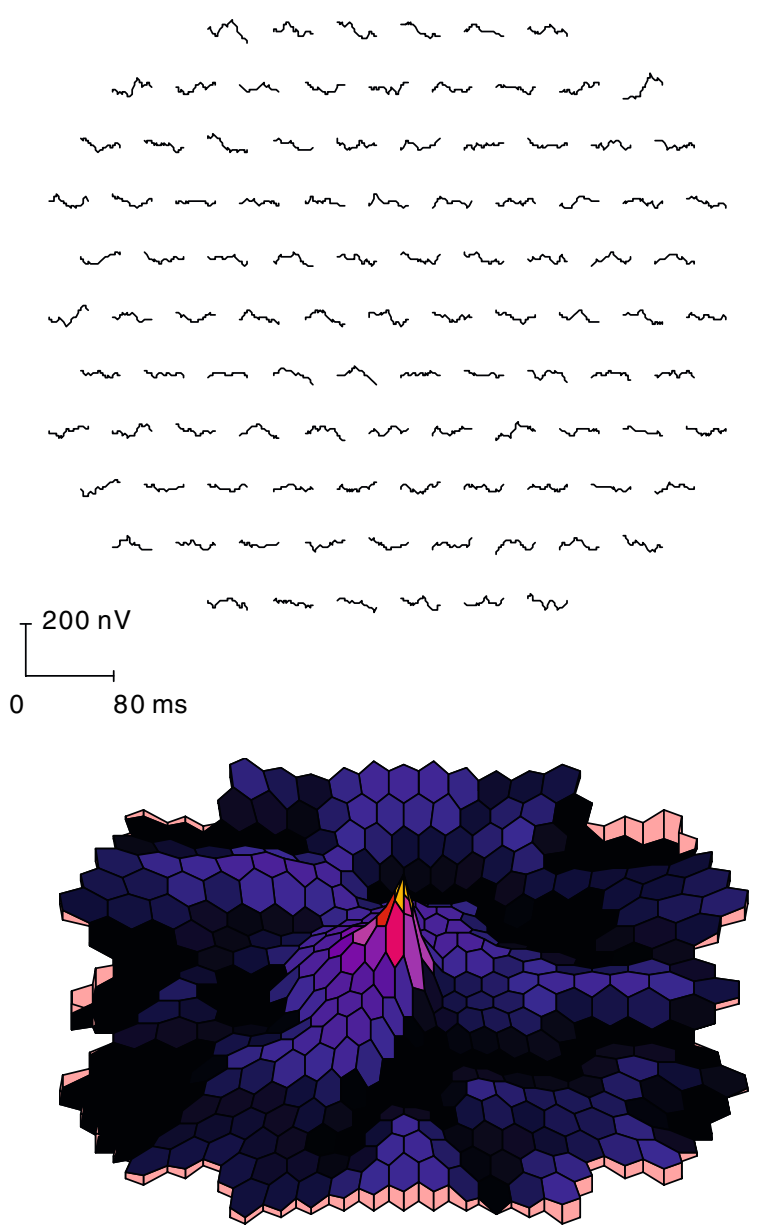

Fig. A4 Weak signals and erroneous central peak. These recordings were obtained from a contact lens electrode placed in a beaker of water. Therefore, there are no mfERG responses in these records. However, the 3-D plot shows a central peak because the noise level is divided by the stimulus area 
determine whether any recognizable waveforms are present.

Averaging and smoothing artifacts areas

Excessive averaging or smoothing of signals will reduce spatial resolution. Severely smoothed records may not reveal small lesions, or show sharp lesion borders. Solution: Avoid unnecessary smoothing, and avoid excessive spatial averaging.

\section{Blind spot}

It is not an artifact that the blind spot is less sharply defined in the mfERG than one might expect. A single stimulus element may not fall totally within the optic disc, so that some response is always obtained. Further, the response attributed to an element falling anywhere in the field is due in part to a response from neighboring regions receiving stray light scattered from this element. The nerve head may reflect more light than other areas of retina, and, thus, the stray light component associated with elements falling on the disc may be disproportionately large.

\section{References}

1. Marmor MF, Holder GE, Seeliger MW, Yamamoto S (2004) International Society for Clinical Electrophysiology of Vision. Standard for clinical electroretinography (2004 update). Doc Ophthalmol 108:107-114

2. Marmor MF, Hood D, Keating D, Kondo M, Seeliger MW, Miyake Y (2003) Guidelines for basic multifocal electroretinography (mfERG). Doc Ophthalmol 106:105-115

3. Holder GE, Brigell M, Hawlina M, Meigen T, Vaegan, Bach M (2007) Standard for pattern electroretinography. Doc Ophthalmol 114:111-116

4. Brigell M, Bach M, Barber C, Moskowitz A, Robson J (2003) Guidelines for calibration of stimulus and recording parameters used in clinical electrophysiology of vision. Doc Ophthalmol 107:185-193 